\title{
Causal Fungus of Side Rot on 'Zesy002' Kiwifruit in Jeju Island, South Korea
}

*Corresponding author

Tel: +82-64-754-3319

Fax: +82-64-725-2351

E-mail:ycjeun@jejunu.ac.kr
Received December 11, 2020

Accepted January 22, 2021

\author{
Magda Ledesma ${ }^{1}$, Yong Ho Shin ${ }^{1}$, Sonia Whiteman ${ }^{2}$, Joy Tyson ${ }^{3}$, Gyoung Hee \\ $\mathrm{Kim}^{4}$, Jeum Kyu Hong ${ }^{5}$, Birgit Zange ${ }^{6}$, and Yong Chull Jeun ${ }^{1,7^{*}}$ \\ ${ }^{1}$ Sustainable Agriculture Research Institute, Jeju National University, Jeju 63243, Korea \\ ${ }^{2} Z$ Zespri International Limited, Mt Maunganui 3149, New Zealand \\ ${ }^{3}$ The New Zealand Institute for Plant \& Food Research Limited (PFR), Auckland 92169, New Zealand \\ ${ }^{4}$ Department of Plant Medicine, Sunchon National University, Suncheon 57922, Korea \\ ${ }^{5}$ Department of Horticultural Science, Gyeongnam National University of Science and Technology \\ (GNTech), Jinju 52725, Korea \\ ${ }^{6}$ Institute of Horticulture, University of Applied Sciences, Weihenstephan-Triesdorf, Freising 85354, \\ Germany \\ ${ }^{7}$ The Research Institute for Subtropical Agriculture and Biotechnology, Jeju National University, Jeju \\ 63604, Korea
}

\begin{abstract}
Since the past few years, dimple rot has become one of the most serious diseases affecting Actinidia chinensis var. chinensis 'Zesy002' kiwifruit in Korea, leading to a low quality and subsequent losses of income. In the current study, the causal pathogen of side rot on 'Zesy002' kiwifruit was identified to be Botryosphaeria dothi$d e a$, a widespread pathogen that could penetrate the fruit directly without wounding. Diaporthe sp., another pathogen causing dimple rot, was isolated from infected kiwifruit. It was not able to infect fruits without wounding. Dimple-rot symptoms are known to be associated with other pathogens from the Pseudocercospora genus. In this study, $B$. dothidea was ratified as the causal disease agent in Jeju Island. To illustrate the pathogenicity of this fungus on 'Zesy002', further studies such as observation of infection structures of fungus may be needed.
\end{abstract}

Keywords: Diagnosis, Dimple rot, Purple blotch, Resistant cultivar, Storage disease

\section{Introduction}

In recent decades, kiwifruit production has been increasing in Asian countries including Korea. Four Actinidia species (A. arguta, A. kolomita, A. polygama, and A.rufa) have been grown naturally in South Korea (Shim and $\mathrm{Ha}, 1999$ ). In the middle 1970s, the 'Hayward' variety from New Zealand was

Research in Plant Disease

pISSN 1598-2262, elSSN 2233-9191

www.online-rpd.org imported to Korea. Later, a yellow-fleshed variety, A. chinensis var. chinensis cultivar 'Hort16A', was introduced to Jeju Island in 2004 (Shim and Ha, 1999). Unfortunately, a bacterial canker outbreak caused by Pseudomonas syringae pv. actinidiae (Psa) vastly occurred in orchards inside and outside New Zealand (Donati et al., 2014). In Korea, Psa was first detected on 'Hayward' variety in 1988 and on 'Hort16A' varity in 2006, resulting in an epidemic on kiwifruit orchards of south provinces, including Jeju Island (Kim et al., 2017a).

In the following years, a search for sweeter varieties with response to previous severe diseases such as canker caused by Psa led to the introduction of a new yellow-fleshed variety

(c) The Korean Society of Plant Pathology

(c) This is an open access article distributed under the terms of the Creative Commons Attribution Non-Commercial License (http://creativecommons.org/licenses/ by-nc/4.0/), which permits unrestricted non-commercial use, distribution, and reproduction in any medium, provided the original work is properly cited. 
of kiwifruit, $A$. chinensis var. chinensis cultivar 'Zesy002', to Korea. This cultivar is grown mostly in the southern part including Jeju Island of South Korea (Zespri International Limited, 2016). However, the growing industry with this new kiwifruit cultivar has brought other challenges such as a storage disease that can reduce profits of kiwifruit farmers in South Korea. Side rot begins as a dimple and a small circular scabbed depression. It is generally developed on the side or near the stem end of the fruit, leading to darkened color from tan to dark brown. Dimples seem to appear during late fruit development. If not recognized during the harvest season, they can develop into fruit rots during cold storage, shortening shelf-life and threatening whole kiwifruit stocks (Pennycook, 1985). These symptoms were similar to those of ripe rot described by Pennycook (1985) known to develop after harvest or removal from cold storage. The causal pathogen of ripe rot is $B$. dothidea which produces lesions only for ripened kiwifruit. This fungal pathogen causes ripe rot for both kiwifruit $A$. deliciosa 'Hayward' and A. chinensis 'Hort16A' varieties (Manning et al., 2003). In Korea, ripe rot caused by $B$. dothidea on A. delicosa 'Hayward' has also been reported (Koh et al., 2005). However, the causal pathogen of side rot on 'Zesy002' kiwifruit has not been known yet. Because side rot occurs not only in late stage, but also in early stage of fruits, the causal pathogen could be different from that causing ripe rot. Furthermore, it has been reported that Pseudocercospora hangzhouensis can cause side or dimple rot symptom on $A$. chinensis 'Hort16A' in Japan and Korea (Yano et al., 2015). Therefore, the exact diagnosis of pathogen on 'Zesy002' kiwifruit causing side rot may be necessary.

In the present study, a diagnosis was carried out for infected 'Zesy002' kiwifruit showing side rot in Jeju orchards. Furthermore, pathogenicities of $B$. dothidea isolates on 'Zesy002' kiwifruit cultivars with or without mechanical wounding were investigated. Similarly, to find the causal fungus on side rot, pathogenicities of both fungi $B$. dothidea and Pseudocercospora sp. were determined by inoculation them onto 'Zesy002' kiwifruit.

\section{Materials and Methods}

\section{Isolation of pathogenic fungi and morphological diag-}

nosis. A total of 64 'Zesy002' kiwifruit showing dimple rot (Fig. 1) or purple blotch were collected from three orchards in Jeju Island of Korea during the 2018 harvest season. These fruits were surface-sterilized by dipping in 1\% (v/v) sodium hypochlorite for $60 \mathrm{sec}$ followed by $70 \%$ (v/v) ethanol for 60 $\mathrm{sec}$, rinsed twice with sterile distilled water for $60 \mathrm{sec}$, and air-dried for $1 \mathrm{hr}$. Kiwifruit skin was peeled back and four segments $\left(1 \mathrm{~cm}^{2}\right)$ were detached from margins of symptomatic underlying tissues and transferred to potato dextrose agar (PDA) plates containing $100 \mu \mathrm{g} / \mathrm{ml}$ of streptomycin sulphate (Sigma Aldrich, St. Louis, MO, USA). After incubation at $28^{\circ} \mathrm{C}$ for 6 days, representative fungal hyphae from each plate were transferred to oatmeal agar medium. For sporulation of the fungus, plates were incubated under light with air supply at $28^{\circ} \mathrm{C}$ for 7 days. Morphological characteristics of fungi were observed under a light microscope (Olympus, Tokyo, Japan).

Molecular identification. Eight isolates from dimple rot and two from purple blotch symptom were selected for molecular identification. Genomic DNA was extracted from approximately $200 \mathrm{mg}$ of fungal hyphae scraped directly from PDA-grown cultures using the cetyltrimethylammonium bromide method (Aamir et al., 2015) with slight modifications. Briefly, hyphae were lyophilized in liquid nitrogen and grinded with mortar and pestle. The powder obtained was placed in a $2 \mu \mathrm{l}$ vial. Then $700 \mu \mathrm{l}$ of lysis buffer (100 mM Tris $\mathrm{HCl}$ [pH 8.0], 50 mM EDTA, 3\% sodium dodecyl sulfate) was added. This mixture was homogenized with a WiseMix Multifunction Vortex Mixer (DAlHAN Scientific, Wonju, Korea) for $20 \mathrm{~min}$ and centrifuged at 13,000 rpm for $10 \mathrm{~min}$. The supernatant was transferred to a fresh $2 \mathrm{ml}$ microcentrifuge tube. Then $2 \mu$ l of RNase A (10 mg/ml) was added and the whole solution was incubated at $37^{\circ} \mathrm{C}$ for $15 \mathrm{~min}$. Later, according to the volume in each vial, equal volume of cleaning buffer (phenol: chloroform: Isoamyl alcohol, 25:24:1) was added. The mixture was homogenized and centrifuged at 13,000 rpm for $10 \mathrm{~min}$. This step was repeated once. The upper aqueous layer was taken in a fresh $1.5 \mathrm{ml}$ micro centrifuge tube and equal volume of $100 \%$ ethanol was added. DNA in this solution was precipitated at $-20^{\circ} \mathrm{C}$ for $30 \mathrm{~min}$ followed by centrifugation at $12,000 \mathrm{rpm}$ for $10 \mathrm{~min}$. The pellet of DNA was washed with $70 \%$ ethanol and centrifuged again at 12,000 rpm for $5 \mathrm{~min}$. Finally, the DNA pellet was air-dried overnight and re-suspended in $1 \times$ TE buffer.

For each sample, $40 \mu \mathrm{l}$ of PCR amplification cocktail contained $2 \mu \mathrm{l}$ of DNA template (5-10 ng/ml), 10 pmol of each forward and reverse primer, $25 \mathrm{mM}$ of $\mathrm{MgCl}, 2.5 \mathrm{mM}$ of each 
A

A
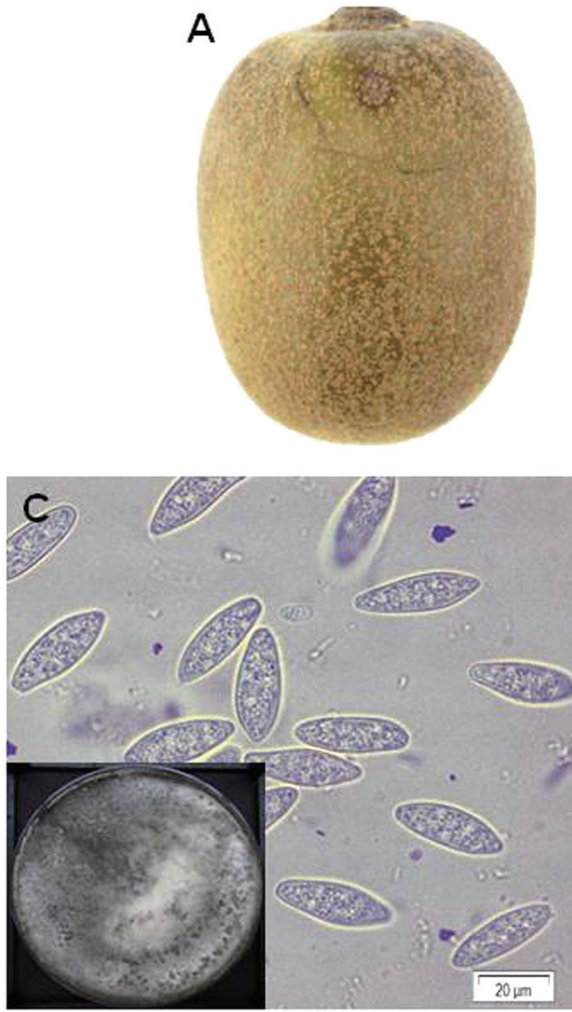

B
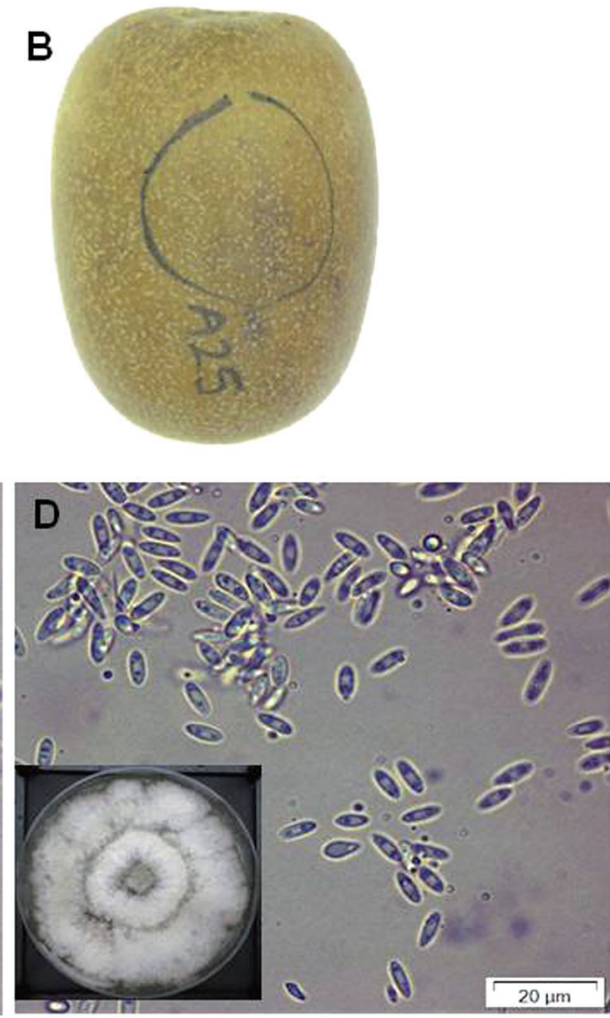

Fig. 1. Typical symptom of dimple rot (A) and purple blotch (B) on 'Zesy002' kiwifruit collected from the orchards in Jeju Island, South Korea during 2018 harvest season. Morphological characteristics of the conidia of isolate C1.1 for type I (C) and A24.3 for type II (D) grown oatmeal agar under light and air supply condition. The square boxes were plates from which the conidia were harvested. The presented photos were taken at 7 days after inoculation. Scale bars $=20 \mu \mathrm{m}(C, D)$.

of the four dNTPs, and 5 units of Taq DNA polymerase. Internal transcribed spacer (ITS) rDNA region was amplified for all selected isolates using universal primers ITS1 (5'-TCCGTAGGTGAACCTGCGG-3') and 4 (5'-TCCTCCGCTTATTGATATGC-3') (White et al., 1990). A set of $B$. dothidea specific primers of FAF (5'-CATCCGCAGCGTGGGAGAACAT-3') and Bt2b (5'-ACCCTCAGTGTAGTGACCCTTGGC-3') were used to reidentify the fungus (Xu et al., 2016). The reaction for ITS was performed on a thermal cycler (Biometra TOne 96G, Analytik Jena AG, Jena, Germany). PCR cycling parameters were: an initial preheat at $94^{\circ} \mathrm{C}$ for $5 \mathrm{~min}$, followed by 35 cycles of denaturation at $94^{\circ} \mathrm{C}$ for $45 \mathrm{~min}$, annealing for $45 \mathrm{sec}$ at $58^{\circ} \mathrm{C}$, and extension at $72^{\circ} \mathrm{C}$ for $1 \mathrm{~min}$, with a final extension stage at $72^{\circ} \mathrm{C}$ for $10 \mathrm{~min}$. In the case of using primer set of FAF/ $\mathrm{Bt} 2 \mathrm{~b}$, the annealing temperature was set at $60^{\circ} \mathrm{C}$.

PCR products were detected by $1 \%$ agarose gel electrophoresis and stained with $0.01 \%$ ethidium bromide for UV light visualization using an UVP transilluminator (UVsolo touch, Analytik Jena AG). Nucleotide sequences of PCR products were determined using an $\mathrm{ABI}$ 3730xI DNA Analyzer (Macrogen Inc., Seoul, Korea). Resulting sequences were compared with sequences in GenBank database using BLAST program of NCBI (http://blast.ncbi.nlm.nih.gov/Blast. cgi) for primary identification.

\section{Pathogenicity test of both isolates and other patho-}

gens. Asymptomatic mature 'Zesy002' kiwifruit were obtained from Zespri-Korea. These kiwifruits were surfacesterilized by dipping in 1\% (v/v) sodium hypochlorite for 60 sec followed by $70 \%(\mathrm{v} / \mathrm{v})$ ethanol for $60 \mathrm{sec}$, rinsed twice with sterile distilled water for $60 \mathrm{sec}$, and air-dried for $1 \mathrm{hr}$. Unwounded and wounded fruits were used. For preparing wounded fruits, kiwifruit were punctured 2-3 mm deep on the upper part with a sterile wood stick right before fungal inoculation.

Representative cultures of both fungal types (isolate C1.1 for Type I and isolate A24.3 for type II) (Supplementary Table 1) were used as inoculum. Several Pseudocercospora species 
have been previously reported to be commonly associated with this kind of side-rot disorder in other kiwifruit varieties (Yano et al., 2015). Thus, Pseudocercospora sp. isolate obtained from the Department of Plant Medicine of Sunchon National University was added as an inoculum. These fungi were transferred into PDA medium and incubated under aerobic conditions with 5,000 lux at $28^{\circ} \mathrm{C}$ for 7 days. Agar plugs with a size of $5 \mathrm{~mm}$ in diameter were detached from the PDA medium and inoculated onto wounded and unwounded kiwifruit. PDA plugs without fungus were used as negative controls. These inoculated fruits were placed in baskets and incubated at $28^{\circ} \mathrm{C}$ with $95 \%$ humidity under no light for 7 days. These experiments were repeated three times with temporal separation. Three replications were carried out in every experiment.

Data analysis. For all experiments, data were analyzed with Duncan's multiple range test using statistical analysis system (SAS) program (version 9.0, SAS Institute Inc., Cary, NC, USA). Statistical significance was considered at $P<0.05$.

\section{Results}

Isolation of pathogenic fungi and morphological diagnosis. A total number of 64 isolates from 61 fruits were obtained (Supplementary Table 1). Among them, most fungal isolates showed phenotypical characteristics similar to those of the main phenotype "Type I" presented principally in kiwifruit with dimple rot symptoms (Fig. 1A). The other phenotype "Type II" was less frequent, although it was also recurrent in kiwifruit with purple blotch symptoms (Fig. 1B).

The conidia of isolate type I showed morphological characteristics similar to those of $B$. dothidea described in other kiwifruit cultivars (Tyson, 2018; Zhou et al., 2015). Furthermore, this fungus has been reported to be the major pathogen associated with postharvest rot on kiwifruit in Korea (Koh et

Table 1. Identification of isolates form different orchards in Jeju Island by BLAST

\begin{tabular}{|c|c|c|c|c|c|}
\hline Isolates & Symptom & Primer & Accession no. & Description & Identity (\%) \\
\hline \multirow[t]{2}{*}{ A2.1 } & Dimple & ITS4 & KX610122.1 & Botryosphaeria dothidea & 98.3 \\
\hline & & FAF & KX306959.1 & B. dothidea & 100 \\
\hline \multirow[t]{2}{*}{ A3.2 } & Dimple & ITS4 & JX179253.1 & B. dothidea & 98.6 \\
\hline & & FAF & HQ529720.1 & B. dothidea & 100 \\
\hline \multirow[t]{2}{*}{ A4.2 } & Dimple & ITS4 & JQ936677.1 & B. dothidea & 100 \\
\hline & & FAF & KP183132.1 & B. dothidea & 100 \\
\hline \multirow[t]{2}{*}{ A13.4 } & Dimple & ITS4 & JX179253.1 & B. dothidea & 99.7 \\
\hline & & FAF & KP183132.1 & B. dothidea & 99.6 \\
\hline \multirow[t]{2}{*}{ B6.2 } & Dimple & ITS4 & JX179253.1 & B. dothidea & 96.3 \\
\hline & & FAF & KP183130.1 & B. dothidea & 98.3 \\
\hline \multirow[t]{2}{*}{ C1.1 } & Dimple & ITS4 & JX179253.1 & B. dothidea & 99.1 \\
\hline & & FAF & KP183132.1 & B. dothidea & 99.3 \\
\hline \multirow[t]{2}{*}{ C19.1 } & Dimple & ITS4 & JQ936677.1 & B. dothidea & 99.0 \\
\hline & & FAF & KP183129.1 & B. dothidea & 99.0 \\
\hline \multirow[t]{2}{*}{ A15.1 } & Dimple & ITS4 & KC145875.1 & Diaporthe sp. & 98.8 \\
\hline & & FAF & - & $\mathrm{nb}$ & - \\
\hline \multirow[t]{2}{*}{ A22.1 } & Purple blotch & ITS4 & KJ609017.1 & D. phaseolorum & 99.1 \\
\hline & & FAF & - & $\mathrm{nb}$ & - \\
\hline \multirow[t]{2}{*}{ A24.3 } & Purple blotch & ITS4 & KC145874.1 & Diaporthe sp. & 99.1 \\
\hline & & FAF & - & $\mathrm{nb}$ & - \\
\hline
\end{tabular}

nb, no band by PCR. 


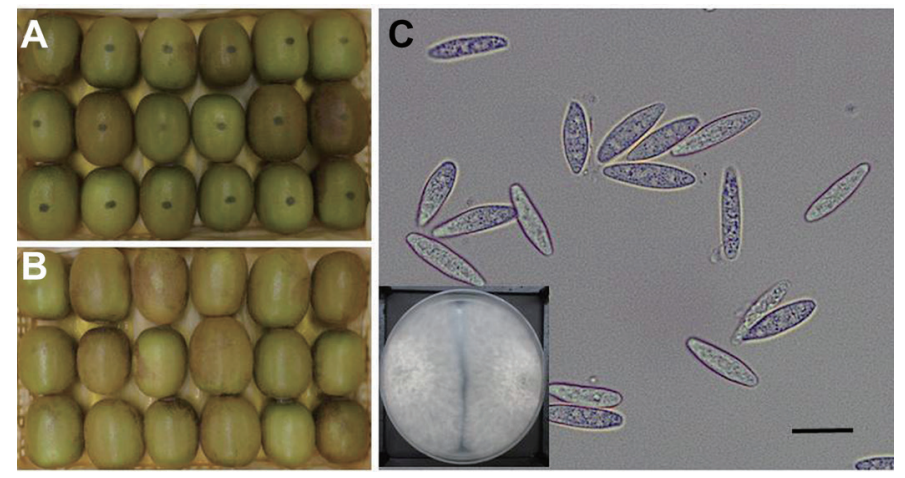

Fig. 2. Side rot symptom on the 'Zesy002' kiwifruit after artificially inoculated with the conidial suspension of isolate $I B$. dothidea with concentration of $1 \times 10^{5}$ conidia/ml $(A)$. On the negative control treated with sterilized $\mathrm{H}_{2} \mathrm{O}$ showed no symptom (B). The presented photos were taken at 7 days after inoculation. Morphological characteristics of the fungal conidia re-isolated from infected kiwifruit (C) which was same with those of first isolated fungus. The presented small square in the down was plate incubated of the re-isolate on PDA for 7 days. Scale bar $=20 \mu \mathrm{m}$ (C).

al., 2003; Kwon et al., 2011). Based on these morphological observations, this type I isolate was suspected as B. dothidea whose conidia were oval to fusoid in shape, aseptate, measuring $7 \pm 1 \mu \mathrm{m}$ in width and $34 \pm 3 \mu \mathrm{m}$ in length. Morphological characteristics of another isolate type II were similar to those of Diaporthe citri (Tyson, 2018; Zhou et al., 2015) whose a-conidia were oval to fusoid in shape, aseptate, measuring $3 \pm 1 \mu \mathrm{m}$ in width and $7 \pm 2 \mu \mathrm{m}$ in length. They have also been found on harvested kiwifruit in Korea (Koh et al., 2003; Kwon et al., 2011).

Molecular identification. Total DNAs were extracted from all 10 selected isolates for PCR. By using ITS primers, total DNAs of all isolates causing dimple rot and purple blotch were amplified. After sequencing, BLASTn showed that all isolates causing dimple rot shared $96-100 \%$ sequence identities with $B$. dothidea strains except for isolate A15.1 (Table 1). Two isolates $A 22.1$ and $A 24.3$ causing purple blotch were identified as $D$. phaseolorum and Diaporthe sp., respectively (Table 1). These results nearly coincided with results of morphological identification (Table 1, Fig. 2, Supplementary Table 1). Using a specific primer set of FAF and $B t 2 b$ for $B$. dothidea, DNA sequences of isolates causing dimple rot were amplified to have a size of $322 \mathrm{bp}$, but not isolates causing purple blotch (Table 1, Fig. 3). As expected, BLASTn analysis

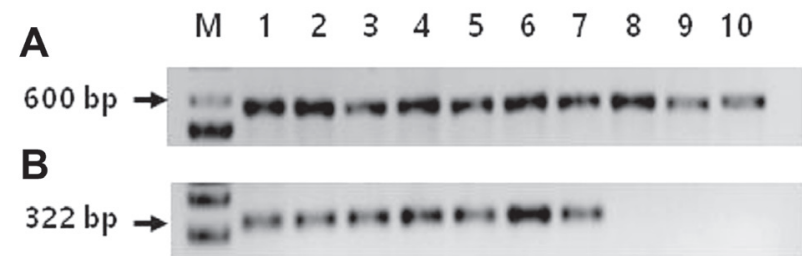

Fig. 3. Gel electrophoresis of PCR-amplified internal transcript spacer (ITS) regions from the isolates using universal primers (A) and using specific primers for $B$. dothidae FAF and $B t 2 b$ (B). $M, 1$ kb ladder (iNtRON Bio); lane 1 (A2.1), lane 2 (A3.2), lane 3 (A4.2), lane 4 (A13.4), lane 5 (B6.2), lane 6 (C1.1), lane 7 (C7.2), and lane 8 (A15.1) were isolates from dimple rot and lane 9 (A22.1) and lane 10 (A24.3) from purple blotch.

revealed that the amplified gene shared $98-100 \%$ sequence identities with B. dothidea (Table 1).

Pathogenicity tests of both isolates and other pathogens. Typical symptoms of dimple rot were observed for wounded 'Zesy002' kiwifruit inoculated with type I isolate $B$. dothidea (Fig. 4A). Unexpectedly, no purple blotch was observed. On the other hand, symptoms like dimple rot were found for wounded kiwifruit inoculated with type II isolate Diaporthe sp. (Fig. 4B). However, Pseudocercospora sp. did not cause any symptom on wounded kiwifruit (Fig. 4C), indicating that 'Zesy002' kiwifruit might not be a host of this fungus. On wounded fruits without fungal inoculation, no symptoms were found (Fig. 4D).

Type I isolate $B$. dothidea successfully infected unwounded kiwifruit (Fig. 4E), indicating their pathogenicity to Gold 3 kiwifruit in nature. However, type II isolate Diaporthe sp. could not infect kiwifruit (Fig. 4F), indicated that type II isolate could not infect Gold 3 kiwifruit by itself. Similarly, symptoms were not observed on unwounded 'Zesy002' kiwifruit inoculated with Pseudocercospora sp. (Fig. 4G), meaning that Pseudocercospora sp. might not be the major pathogen for dimple rot. On unwounded kiwifruit, no symptom was found (Fig. 4H).

\section{Discussion}

Postharvest dimple rot constitutes a serious challenge to kiwifruit production in Jeju Island, Korea. Pathogenic fungi associated with this disease on 'Zesy002' kiwifruit need to be identified in order to decrease economical loss of kiwifruit farms. Dimple-like and purple blotch lesions were recognized (Whiteman, 2018) in orchard or storage during 2018-2019 

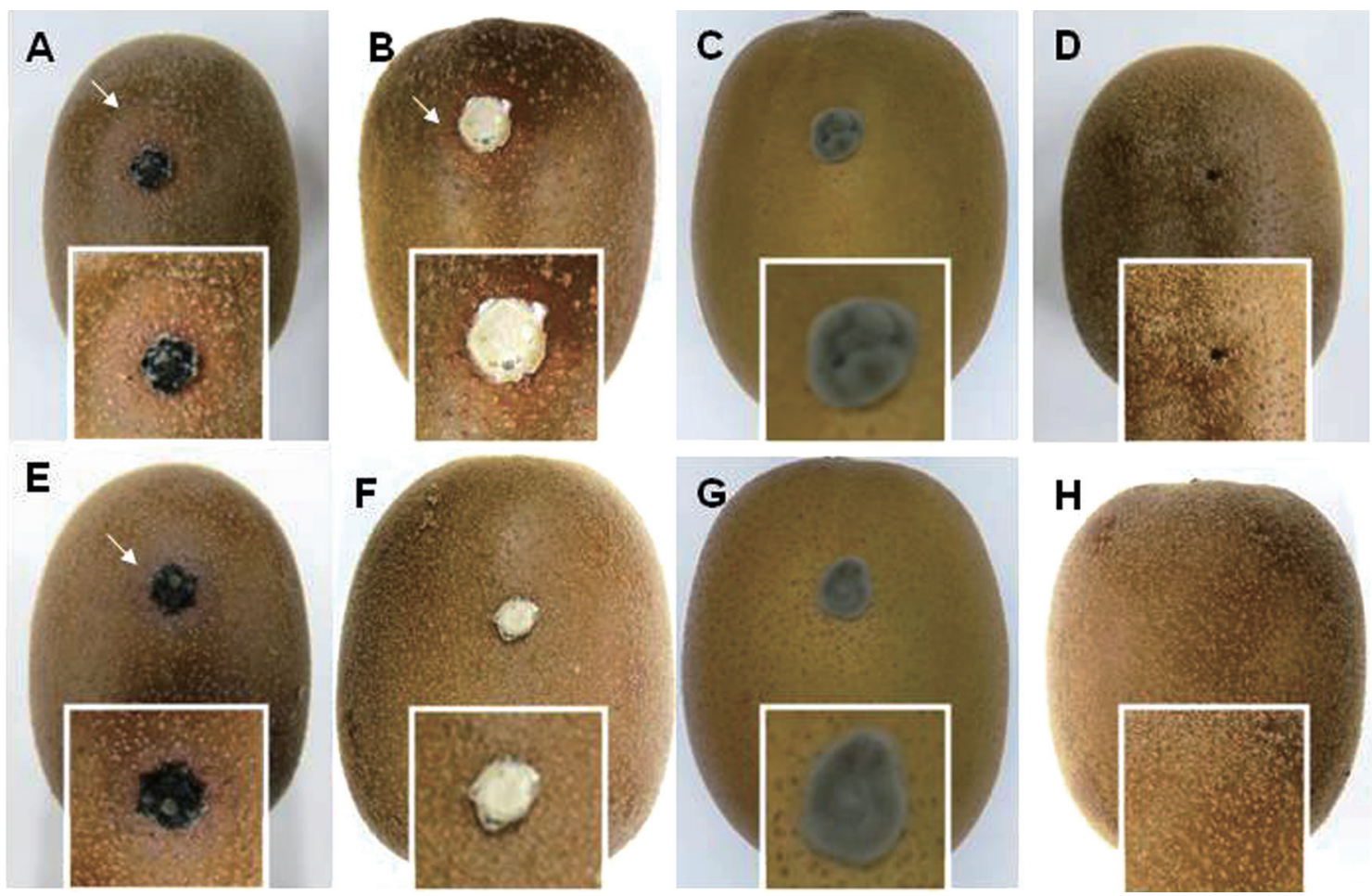

Fig. 4. External symptoms of dimple rot on artificially inoculated on the wounded/unwounded 'Zesy002' kiwifruit with the type I isolate $B$. dothidea (A, E), type II isolate Diaporthe sp. (B, F), Pseudocercospora sp. (C, G), and sterile agar plug $(D, H)$, respectively. The presented photos were taken at 7 days after inoculation. The square boxes represented the magnified of the inoculated part. The arrow showed the symptom of dimple rot.

harvest seasons on 150 'Zesy002' kiwifruit from 13 greenhouses around Jeju Island. Dimple symptoms cause small scabbed depressions on the side or near the stem end of the kiwifruit. They can develop internally into a side rot. Purple blotch symptoms cause darker areas of the fruit of a purplish color. In some cases, the fruit skin looks dried and shrunken. Identical dimple signs have been reported in several other kiwifruit cultivars in Korea, China, New Zealand, and other countries (Kim et al., 2017b; Koh et al., 2003; Kwon et al., 2011; Manning et al., 2003; Zhou et al., 2015), but not in 'Zesy002' kiwifruit. In the present work, these symptoms were excised and cultured, resulting in 64 isolates, from which 95\% of them showed similar morphological characteristics (Fig. 2). Isolate type I from kiwifruit showed morphological characteristics similar to those of fungus on other kiwifruit cultivars described before. It was identified as Botryosphaeria dothidea (Tyson, 2018; Zhou et al., 2015). Their abundant gray to olive aerial mycelia and the hyaline unicellular a-conidia of oval shape matched with the Ascomycota genus, Botryosphaeria. DNA sequence identification of a sample of 50 isolates shared $100 \%$ identities with B. dothidea strains (Fig. 3). Fur- thermore, this fungus has been reported to be the major pathogen associated with postharvest rot on kiwifruit in Korea with incidence of $83.3 \%$ to $95.4 \%$ (Koh et al., 2003; Kwon et al., 2011). Based on our inoculation experiments and morphological observations, this type I isolate was identified as $B$. dothidea.

Isolate type II seemed to be Diaporthe citri reported on harvest kiwifruit with a frequency of $4.6 \%$ to $11.9 \%$ in Korea (Koh et al., 2003; Kwon et al., 2011). It has been reported that this fungus could not infect kiwifruit without wound (Li et al. 2017), similar to finding of the present study. This result suggests that $D$. citri may be saprophytic in kiwifruit. It needs wound by pathogenic fungus such as $B$. dothidea to infect kiwifruit.

B. dothidea is a globally widespread pathogen associated with dieback, canker, and severe fruit rots. It has an extensive host range (Beckman and Reilly, 2005; Milholland, 1972; Pitt et al., 2010; Tang et al., 2012). Through pathogenicity testing, it was found that $B$. dothidea could penetrate the fruit directly without wounding. However, the presence of a wound exacerbates this disease, accelerating its development and 
intensifying its severity (Li et al., 2017; Zhou et al., 2015). After re-isolation from artificially inoculated kiwifruit, the same symptoms in the fruit as well as the same cultural behavior, morphology, and DNA sequence identity were found (Fig. 3), thus confirming Koch's Postulates and ratifying $B$. dothidea as a causal agent of postharvest dimple rot on 'Zesy002' kiwifruit in Jeju Island.

In South Korea, yellow-fleshed varieties have shown to be less resistant to postharvest rots than green fleshed ones (Kwon et al., 2011). Ploidy level might play a fundamental role in breeding resistance against bacterial canker caused by Psa (Tahir et al., 2019), a pathogenic bacterium that can lead to severe crop loss. Therefore, evaluation of susceptibilities to B. dothidea among 'Hort16A' (diploid), 'Zesy002' (tetraploid) and 'Hayward' (hexaploid) varieties may provide insights about a possible relationship between predisposition to dimple rot and ploidy level. However, dimple-rot susceptibility might not follow the ploidy hypothesis applied for Psa. Additional research is needed to find candidate alleles for susceptibility and resistance to dimple-rot disease.

On the other hand, solitary dimples on the side of kiwifruit have been previously reported as characteristic symptoms caused by Mycosphaerellaceae pathogens Pseudocercospora actinidiae and Pseudocercospora hangzhouensis (Yano et al., 2015). This possibility was explored by comparing the pathogenicity capacity of Pseudocercospora sp. with that of $B$. dothidea under the same conditions. While $B$. dothidea was able to penetrate the unwounded fruit skin, this strain of Pseudocercospora sp. did not result in any symptoms. It was not re-isolated from any inoculated kiwifruit, indicating that Pseudocercospora sp. might not be the major pathogen for this form of dimple rot in 'Zesy002'. These results open new possibilities for integral management of dimple rot disease, in which protecting the fruit during crucial developmental stages and designing different storage conditions might better complement control strategies for 'Zesy002' kiwifruit disease. Further experimentation regarding $B$. dothidea life cycle, overwintering of secondary inoculum, and the possibility of symbiotic relations with fruit feeding insects may be needed to develop a successful strategy for 'Zesy002' kiwifruit protection in Jeju Island.

\section{Conflicts of Interest}

The current study was financially supported by Zespri Group
Limited. Sonia Whiteman is employee of Zespri International Limited. All other authors have no competing interests.

\section{Acknowledgments}

This work was supported by Zespri Group Limited, New Zealand through a research and development service program and a research grant of Jeju National University in 2019.

\section{Electronic Supplementary Material}

Supplementary materials are available at Research in Plant Disease website (http://www.online-rpd.org/).

\section{References}

Aamir, S., Sutar, S., Singh, S. K. and Baghela, A. 2015. A rapid and efficient method of fungal genomic DNA extraction, suitable for PCR based molecular methods. Plant Pathol. Quar. 5: 74-81.

Beckman, T. G. and Reilly, C. C. 2005. Relative susceptibility of peach cultivars to fungal gummosis (Botryosphaeria dothidea). J. Am. Pomol. Soc. 59: 111-116.

Donati, I., Buriani, G., Cellini, A., Mauri, S., Costa, G. and Spinelli, F. 2014. New insights on the bacterial canker of kiwifruit (Pseudomonas syringae pv. actinidiae). J. Berry Res. 4: 53-67.

Kim, G. H., Jung, J. S. and Koh, Y. J. 2017a. Occurrence and epidemics of bacterial canker of kiwifruit in Korea. Plant Pathol. J. 33: 351361.

Kim, G. H., Kim, D. R., Park, S.-Y., Lee, Y. S., Jung, J. S. and Koh, Y. J. 2017b. Incidence rates of major diseases of kiwiberry in 2015 and 2016. Plant Pathol. J. 33:434-439.

Koh, Y. J., Hur, J.-S. and Jung, J. S. 2005. Postharvest fruit rots of kiwifruit (Actinidia deliciosa) in Korea. N. Z. J. Crop Hortic. Sci. 33: 303310.

Koh, Y. J., Lee, J. G., Lee, D. H. and Hur, J.-S. 2003. Botryosphaeria dothidea, the causal organism of ripe rot of kiwifruit (Actinidia deliciosa) in Korea. Plant Pathol. J. 19: 227-230.

Kwon, S. Y., Kim, G. H., Koh, Y. J., Lee, Y. S., Sohn, S. H., Kim, S.-C. et al. 2011. Incidence rates of postharvest fruit rots and detection rates of their pathogens on new kiwifruit cultivars bred in Korea. Korean J. Plant Res. 24: 599-603.

Li, L., Chen, M., Zhang, S. and Zhong, C. 2017. Isolation and identification of pathogenic fungi causing postharvest fruit rot of kiwifruit (Actinidia chinensis) in China. J. Phytopathol. 165: 782-790.

Manning, M. A., Meier, X., Olsen, T. L. and Johnston, P. R. 2003. Fungi associated with fruit rots of Actinidia chinensis 'Hort16A' in New Zealand. N. Z. J. Crop Hortic. Sci. 31:315-324.

Milholland, R. D. 1972. Histopathology and Pathogenicity of Botryosphaeria dothidea on blueberry stems. Phytopathology 62: 654- 
660.

Pennycook, S. R. 1985. Fungal fruit rots of Actinidia deliciosa (kiwifruit). N. Z. J. Exp. Agric. 13: 289-299.

Pitt, W. M, Huang, R., Steel, C. C. and Savocchia, S. 2010. Identification, distribution and current taxonomy of Botryosphaeriaceae species associated with grapevine decline in New South Wales and South Australia. Aust. J. Grape Wine Res. 16: 258-271.

Shim, K.-K. and Ha, Y.-M. 1999. Kiwifruit production and research in Korea. Acta Hortic. 498: 127-132.

Tahir, J., Hoyte, S., Bassett, H., Brendolise, C., Chatterjee, A., Templeton, $\mathrm{K}$. et al. 2019. Multiple quantitative trait loci contribute to resistance to bacterial canker incited by Pseudomonas syringae pv. actinidiae in kiwifruit (Actinidia chinensis). Hortic. Res. 6: 101.

Tang, W., Ding, Z., Zhou, Z. Q., Wang, Y. Z. and Guo, L. Y. 2012. Phylogenetic and pathogenic analyses show that the causal agent of apple ring rot in China is Botryosphaeria dothidea. Plant Dis. 96: 486-496.

Tyson, J. L. 2018. Identification of Side Rots in Korean Actinidia chinensis var. chinensis "'Zesy002"' (Gold3) Fruit. Zespri Group Limited Report No. Gl1981-30-a. Zespri International Limited, Mount Maunganui, New Zealand. pp. 7-8.

White, T. J., Bruns, T., Lee, S. and Taylor, J. 1990. Amplification and direct sequencing of fungal ribosomal RNA genes for phylogenetics. In: PCR Protocols: A Guide to Methods and Applications, eds. by M. A. Innis, D. H. Gelfand, J. J. Sninsky and T. J. White, pp. 315-322. Academic Press, San Diego, CA, USA.

Whiteman, S. 2018. Korean Side Rot Management: Programme Outline. Zespri International Limited, Mount Maunganui, New Zealand. pp. 1-4.

Xu, C.-N., Zhang, H.-J., Chi, F.-M., Ji, Z.-R., Dong, Q.-L., Cao, K.-Q. et al. 2016. Species-specific PCR-based assays for identification and detection of Botryosphaeriaceae species causing stem blight on blueberry in China. J. Integr. Agric. 15: 573-579.

Yano, T., Nakashima, C., Nakahama, M., Kimura, K., Tsuneoka, H., Shimizu, S. et al. 2015. Features and the seasonal variations in emergence of two side-rot symptoms on "'Hort16A"' kiwifruit. Acta Hortic. 1096: 389-396.

Zespri International Limited. 2016. Making Life Delicious: Annual Review 2015/16. Zespri International Limited, Mount Maunganui, New Zealand. 14 pp.

Zhou, Y., Gong, G., Cui, Y., Zhang, D., Chang, X., Hu, R. et al. 2015. Identification of Botryosphaeriaceae species causing kiwifruit rot in Sichuan province, China. Plant Dis. 99: 699-708. 